CHAPTER 10

\title{
NETWORK AND SPACE OBSERVATIONS OF SOLAR AND STELLAR OSCILLATIONS
}




\title{
SPACE OBSERVATIONS OF SOLAR AND STELLAR OSCILLATIONS
}

\author{
Robert W. Noyes . \\ Havard-Smithsonian Center for Astrophysics \\ 60 Garden Street \\ Cambridge, MA 02138 USA
}

\begin{abstract}
Space observations offer many advantages for helio- and asteroseismology, due principally to the absence in space of transparency fluctuations, image blurring, and absorption by the Earth's atmosphere, and to the possibility of long-term continuous observations by a single spaceborne instrument. Helioseismology from space has already shown that stars produce a measurable oscillation signal through photometric variations at the micromagnitude level; this leads to the near-term possibility of simple spaceborne instruments for both helio- and asteroseismology. For the Sun, where photon flux is not a serious problem, doppler analyzer instruments also show great promise. A number of space investigations in helio- and asteroseismology presently under definition or development are briefly discussed.
\end{abstract}

\section{INTRODUCTION}

Space observations are complex and expensive, and should be undertaken only if the desired information is both extremely important and unobtainable from the ground. While the importance of observations of solar and stellar oscillations is not in question, the need for observing them from space is not so obvious. Usually space investigations are justified either because the spectral emissions under study do not penetrate to the ground, because sub-arcsec imaging is required, or (in the case of solar system exploration) because in situ sampling or close-up viewing is required. Helio- and asteroseismology pose none of these requirements. Therefore we shall first explore the detailed rationale that drives us to consider space an appropriate environment for work in this area. In the subsequent two sections we will review current ideas for instrumentation for space observations of solar and stellar oscillations respectively.

There are several clear advantages of a location in space rather than on the ground for observations of solar or stellar oscillations:

1. Observations from space eliminate degradation of data by atmospheric inhomogeneities. Space observations permit studying solar or stellar oscillations through photometric variations of broadband flux from the star, whereas the same observations from the earth are rendered useless by time variations of atmospheric transparency. In addition, for the solar case where light is collected over a $1 / 2$-degree disk, transmission gradients across the disk further degrade ground-based photometric accuracy, both for spatially-resolved and unresolved (sun-as-a-star) observations. Finally, image blurring ("seeing") at typical ground-based locations averages several arcseconds. (The relevant 
quantity is the average seeing over the many-day length of an observation sequence, rather than the rarely-obtained best seeing.) Thus solar observations at very high $l$ are severely compromised. Not only does image blurring decrease the signal-to-noise ratio, but it also mixes modes with adjacent values of $l$.

\section{Observations from space can take advantage of complete transparency at all} wavelengths. Thus doppler shifts of solar or stellar absorption lines may be measured without contamination from terrestrial absorption lines. Also, observations are possible in ultraviolet or infrared regions inaccessible from the ground. The photometric variation due to oscillations should be greater in the ultraviolet than in the visible, thus increasing the signal-to-noise ratio for some observations.

\section{Continuous, uninterrupted observations are possible from some locations in space.}

From the ground, such observations are difficult at best, and in practice some interruptions will be inevitable. In principle, continuous observations are possible from the south pole, but only during a short period of the year; also they are logistically formidable, and interruptions due to clouds are to be expected. Networks of observations at more moderate latitudes are feasible (Fossat 1986) but (especially for solar observations at high $l$ interleaving of the observations from different instruments will present great challenges.

4. For some orbits, the velocity of the instrument with respect to the Sun will vary to a much less degree than at most ground-based sites. This can eliminate problems of instrumental drift due to changing instrument-Sun velocity.

\section{The ability to observe from space in principle allows "stereoscopic" observations of} solar oscillations. As an example, solar oscillation instruments could be spaced along the ecliptic to observe oscillations at all longitudes around the Sun simultaneously.

The first advantage, that degradation by atmospheric variations is eliminated, is more important for solar than for stellar oscillation observations, both because atmospheric image blurring compromises high- $l$ solar data, and because transparency gradients across the extended image degrade the low-l solar data. Stellar oscillation observations are immune to both of these problems, so the advantages of space for asteroseismology are not so immediately obvious. For point sources the effect of atamospheric transparency variations can in principle be overcome by differential spectral measurements (such as doppler shift observations, or differential chromospheric and photospheric spectrophotometry), just as is done for the solar case, or for the few previous stellar oscillation measurement attempts. Thus, a network of ground-based telescopes for stellar studies (to obtain long continuous data sets) would seem a very attractive alternative for asteroseismology. However, unlike the solar case where photon flux is not an overriding problem, here the atmosphere exacts a large price by forcing the use of differential narrowband spectroscopy rather than allowing the broadband photometry which is possible only from space. This price translates in to the cost to devote rather large aperture telescopes to the observations; a network of several ground-based telescopes each of several meters aperture may exact a price comparable to or larger than a small dedicated stellar photometric instrument in space.

As helio- and astero-seismology begins to tackle harder and harder problems (such as the discovery, identification, and detailed analysis of g-modes on the Sun, the measurement of rotational splittings of stellar p-modes, or time variations of oscillation modes) the advantages of a very stable, long-lived, continuously operating instrument in space become even clearer. An illustration of this point is provided by the ACRIM instrument 
on SMM. Although the ACRIM data have very much lower signal-to-noise ratio than many ground-based helioseismology instruments (principally because ACRIM was not designed with helioseismology in mind), because of its long lifetime in orbit, it has obtained rather precise frequencies for the low-degree solar p-inodes, as well as evidence for their variation over time, presumably associated with the solar magnetic activity cycle. It may be anticipated that with instrumentation expressly designed for the purpose, space helio- and astero-seismology will ultimately far exceed in scientific return the best that can ever be obtained from the ground.

\section{SPACE HELIOSEISMOLOGY INVESTIGATIONS}

\subsection{The ACRIM Instrument on SMM and its successors}

The Active Cavity Radiometer Irradiance Monitor (ACRIM) records the bolometric solar flux, or irradiance. It is described in detail by Willson (1979) and Willson and Hudson (1981). It operated nearly continuously from 18 February 1980 to 1 December 1980 and again from 1 May 1984 to the present. While designed to measure the solar irradiance and its variation on active-region time scales, it has sufficient sensitivity also to record the micromagnitude variations produced by p-mode oscillations. As already noted, the relatively long continual periods of data acquisition have permitted rather precise determination of p-mode eigenfrequencies (Woodard and Noyes 1985, 1986). These results are suggestive that the internal structure of the sun varies with the magnetic activity cycle in a measurable way; confirmation of the result and its unambiguous association with the activity cycle await continued observations with ACRIM or other helioseismology instruments over coming years.

An important additional result of the ACRIM investigation is the verification that the Sun reflects the existence of p-mode oscillations through photometric variability at the micromagnitude level; the strongest modes have an amplitude $\Delta \mathrm{I} / \mathrm{I} \approx 1.8 \times 10^{-6}$. This immediately opens up the possibility of stellar oscillation observations from space using rather simple instrumentation, as we discuss in part III below.

It is intended (Willson 1986, personal communication) that successors to the ACRIM instrument on SMM will fly on the series of Geophysical Orbiting Environmental Satellites (GOES); these satellites will be in geosynchronous orbit, and thus will experience nearly continuous solar viewing. In addition, instrument upgrades are planned to incorporate a modest degree of angular resolution. Even moderate angular resolution will greatly reduce the complexity of the spectrum of low-degree modes, by permitting separation of prograde and retrograde modes, or tesseral and zonal modes. Such separation could aid in determining, respectively, the rotational profile or the role of magnetic fields in the deep interior. Also it could facilitate the detection and identification of low-order g-modes, which also suffer from confusion because of the great density of modes expected. If $\mathrm{g}$-modes have a larger amplitude in intensity than in velocity, then such space observations, combining photometry with modest angular resolution, may be the best way to study g-modes.

\subsection{Other Solar Irradiance Monitoring Instruments}

C. Fröhlich and collaborators have been developing a series of solar irradiance monitors for monitoring integrated-light solar variability at several wavelengths simultaneously (Fröhlich 1986). All have the goal of measuring solar irradiance variability on a time scale of minutes to months to study properties of the Sun, as well as solar influence on the Earth's climate. They are designed to measure not just total solar irradiance, but 
also spectral irradiance--that is, irradiance in several different spectral bands simultaneously. Spectral irradiance should give additional information on $\mathrm{p}$ - and g-modes beyond that provided by the total irradiance variation; namely the amplitude and phase relationships between spectral irradiance variations in different wavelength bands should give information on the propagation characteristics (e.g. associated temperature fluctuations) of the modes.

The first of the SIM series is the Interplanetary Helioseismology by Irradiance Measurements (IPHIR) experiment expected to be launched on the PHOBOS spacecraft of the USSR, in 1988. The IPHIR experiment contains three spectral channels at 335,500 , and $865 \mathrm{~nm}$, and will provide resolution of better than 1 micromagnitude. The second of the series, the Solar Irradiance Variability (SOVA) experiment, is scheduled to be launched about 1991. This experiment will be part of the EUropean REtrievable CArrier (EURECA) mission, which will stay in orbit for six months and then be retrieved by the shuttle. It will contain two different types of absolute radiometers, plus a spectral irradiance instrument with six spectra channels between 335 and $865 \mathrm{~nm}$. Finally, the third of the series, the Solar Irradiance Monitor (SIM) is expected to be flown on the Solar Oscillations and Heliospheric Observatory (SOHO) spacecraft, scheduled to be launched about 1993, and to remain in operation for at least two years. This would contain three independent absolute radiometers--the two mentioned above plus an ACRIM, and a spectral irradiance monitor with six spectral channels, as above. The SOHO spacecraft and its instrumentation are described in more detail by Domingo (1986).

\subsection{The Solar Velocity Monitor (SVM) on SOHO}

Also baselined on SOHO is a Solar Velocity Monitor; a design study of such an instrument has been carried out by P. Hoyng and collaborators (Hoyng 1986). This instrument is designed to measure solar velocity oscillations in integrated sunlight, with a sensitivity ranging from $10^{3}(\mathrm{~m} / \mathrm{s})^{2} \mathrm{~Hz}^{-1}$ at $100 \mathrm{nHz}$ to $10^{-2}(\mathrm{~m} / \mathrm{s})^{2} \mathrm{~Hz}^{-1}$ at $10 \mathrm{mHz}$. This is well below the best estimates of velocity noise power in the solar continuum (Harvey 1985). The instrument measures the doppler shift of the $769.9 \mathrm{~nm}$ line of neutral potassium, using a resonant scattering spectrometer fed by a $13-\mathrm{cm}$ telescope.

\subsection{The Solar Oscillations Imager (SOI) on SOHO}

This investigation, to be supplied by NASA as one of the U.S. complement of instruments for SOHO, has been the subject of several studies over the past several years. After an initial conceptual study by an international group of scientists (Noyes and Rhodes 1984), a Phase A study has been carried out at the NASA Jet Propulsion Laboratory. The main scientific goal is to obtain resolved velocity oscillation to $l \simeq 700$, using a $1024 \times 1024$ imaging detector; such data are critical for studying the structure and dynamics of the convection zone, as well as the deep interior. The baseline instrument includes a magneto-optical filter (cf. Rhodes et al 1984,1986) feeding a CCD detector. Because images on the red and violet wing of the spectral line are read out once per minute from a one megapixel image, a rather high data rate of about $160 \mathrm{~Kb} / \mathrm{s}$ is required for full data recovery. Such a data flow is anticipated to occur continuously for several periods of two months or more during the SOHO mission, through use of the NASA Deep Space Network. During other times, there will be periods when only much lower data rates are possible; at these-times on-board spatial averaging would be used to select out a subset of the total information for transmission to earth. 


\section{PROPOSED SPACE ASTEROSEISMOLOGY INVESTIGATIONS}

Because of the extreme faintness of stellar sources compared to the Sun, asteroseismology is always photon-starved. On the ground, it is possible to study stellar oscillations using large telescopes, and hence techniques which use only a small fraction of the total available starlight are feasible. These are discussed by Harvey (1986). In space, however, the dedicated use of large aperture telescopes for asteroseismology is not presently a realistic possibility. Therefore, most proposed space investigations have concentrated on broadband photometric studies, which record a large fraction of the stellar spectrum.

Such proposals find their rationale in the success of the ACRIM investigation mentioned earlier. If we assume that other stars behave essentially like the Sun, then the capability to detect photometric variability with an amplitude of $10^{-6}$ should allow measurement of the strongest low-degree modes with $\mathrm{S} / \mathrm{N}$ of about 3 . To obtain such $\mathrm{S} / \mathrm{N}$ then requires the detection of $10^{12}$ photons for a photon noise-limited system. This represents a threshhold for useful measurements; one would of course likt to do considerably better.

Figure 1 gives the integration time required to detect $10^{12}$ photons in broadband visible radiation from dwarf $F, G$, and $K$ stars of various apparent magnitudes. We see that for bright neighborhood dwarfs, with apparent magnitudes less than about 6 , the threshhold of $10^{12}$ photons can be detected in less than 10 days with telescopes of aperture less than $30 \mathrm{~cm}$. However, a star as faint as $\mathrm{V}=12$ would require 100 days integration with a $1-\mathrm{m}$ aperture; this approaches the practical limit for realistic space asteroseismology missions.

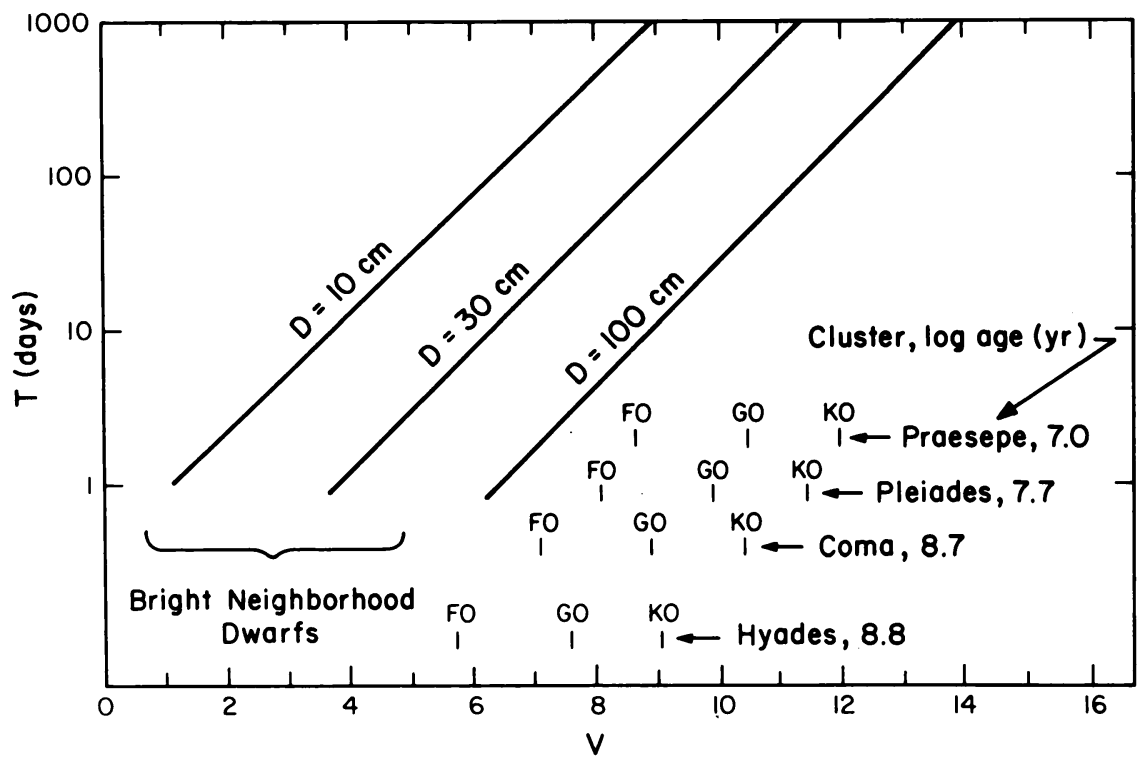

Figure 1. Time to reach $\mathrm{S} / \mathrm{N}=10^{6}$, in broad band (035 to $1 \mu$ ), with 0.12 system efficiency, for various apertures $D$. 


\subsection{Asteroseismology from the Hubble Space Telescope (HST)}

The Hubble Space Telescope (HST) contains a High-Speed Photometer which is capable of measuring photometric variations with precision sufficient for asteroseismology. Soderblom and colleagues (Soderblom 1986) have proposed an asteroseismology investigation of the star $\alpha$ Cen A, using the HST High-Speed Photometer. This star has been selected as the prime target because it has very well-known mass, radius, and effective temperature, and makes an ideal comparison source to the Sun; in addition because of its location it is possible to observe this star for long continuous periods without occultation by the Earth.

\subsection{Asteroseismology from the USSR Vesta Mission}

VESTA is a French-Soviet mission towards Mars and later to the asteroid belt. It is expected to be launched in the 1992-1994 time frame. It has been proposed (Praderie et al 1986) to do limited asteroseismology observations during the cruise phase, which would last 9 months during the journey to Mars and perhaps an additional 4.5 years if there is later travel to the asteroids. The available volume on VESTA for asteroseismology limits the proposed aperture to $5 \mathrm{~cm}$ only. However, this is sufficient to study very bright stars $(\mathrm{V} \leq 4)$. Observations will be made simultaneously in two $1500 \mathrm{~A}$ pass bands, so that oscillations can be studied in different colors. For this system a photon noise of $10^{-6}$ magnitude can be reached in about four months of continuous observations. Thus during the cruise phase to Mars two such stars could be studied, from each of the two spacecraft planned.

\subsection{Asteroseismology from small earth-orbiting telescopes.}

Because the required aperture for asteroseismology of bright neighborhood stars is so modest (Figure 1), it should be possible to piggy-back a small telescope on earth-orbiting platforms at modest expense, and monitor a single bright star for several months at a time. While this approach suffers compared to that described immediately below in that only one star can be studied at a time, because of its simplicity it may be a good method for early exploration of the potential of asteroseismology from space. Studies of such systems are underway by the author and colleagues in the USA and by Praderie and collaborators in Europe (Praderie, personal communication).

\subsection{Asteroseismology of Star Clusters}

Asteroseismology requires an extended period of observation (i.e. months) for any star under study. However, it is necessary to measure the oscillation spectrum of many stars in order to make real progress in understanding how interior structure depends on properties such as mass, age, or rotation rate. If stars of interest are measured one by one, the time required to carry out an optimum study becomes inordinately long. Hence it has been proposed (Hudson et al, 1986) to image a relatively dense star field, such as an open cluster, on a two-dimensional detector array and measuring the oscillation spectrum of a number of stars simultaneously. This is a clear case where observations from space are an advantage, since the possibility of measuring photometric rather than spectroscopic variations over a two-dimensional image considerably simplifies the instrumentation; in addition it permits observations at a much fainter limiting magnitude for a given aperture telescope than does spectroscopy. The proposal calls for a 1-m Schmidt telescope, mounted on an Explorer satellite, to image a star field upon a CCD detector. Such instrumentation would permit the study of oscillations in stars in several open 
clusters, such as the Hyades, (age $7 \times 10^{8} \mathrm{yrs}$ ), the Pleiades (age $5 \times 10^{7} \mathrm{yrs}$ ), or Perseus (age $1 \times 10^{7}$ years); see Figure 1 . By studying coeval star groups of different ages, one may also hope to obtain important information about evolution of interior structure in lower main sequence stars.

\section{SUMMARY AND CONCLUSIONS}

The above discussion includes initiatives known to the author to be under way or at least under serious consideration at the time of the Symposium. The list is doubtless incomplete, for the advantages of space for helio- and asteroseismology are so evident that a great many new ideas are presently being explored.

Unfortunately, other than the ACRIM experiment no results from space are available for comparison of alternative observational approaches. However, even without existing space data, ground observations indicate that for imaged observations of the Sun, doppler observations such as proposed for the SVM or SOI on SOHO are the most promising direction for space observations (Harvey 1985). As far as integrated-light helioseismology, and asteroseismology, go, we may at least build on the ACRIM results, which clearly show the feasibility of studying stellar oscillations by means of radiometry or broad-band photometry. Because of the relative simplicity of photometers plus the relatively large light grasp from broad-band observations, this demonstration has dominated planning, at least for the near term. Even very small telescopes, such as those that could be accomodated on interplanetary probes, should be able to obtain useful data.

In the longer term, it is possible that doppler measurements of stellar oscillations, employing large space telescopes, will become feasible; here as for the Sun one may anticipate a net gain in signal-to- noise because of the relatively higher intrinsic $\mathrm{S} / \mathrm{N}$ ratio of the stellar oscillation signal. However, the first space reconnaisance of stellar oscillations is likely to be by broadband photometry, for reasons of instrumental simplicity and economy.

Finally, we note the gain from imaging stars in clusters in order to collect observational data from a number of stars at once; the advantage of collecting data from a number of stars at one time, plus the likely common age of the stars under study, may outweigh the greater instrumental complexity. Clusters of interest are quite a bit fainter than the bright field stars of interest (Figure 1), so larger apertures will be needed than for individual studies of bright field stars.

\section{References}

Domingo, V. 1986, these proceedings.

Fossat, E. 1986, these proceedings.

Fröhlich 1986, personal communicaton; see also "SOHO: Report on the Phase A Study," ESA Report $\operatorname{SCI}(85) 7,1985$, p. 71.

Harvey, J.W. 1985, in "Future Missions in Solar, Heliospheric, and Space Plasma Physics" (ESA SP-235), p. 199.

Harvey, J.W. 1986, these proceedings.

Hoyng, P. 1986, personal communication; see also "SOHO: Report on the Phase A Study," ESA Report SCI(85) 7, 1985, p. 70.

Hudson, H., Brown, T., Christensen-Dalsgaard, J., Cox, A.N., Demarque, P., Harvey, J., McGraw, J., Noyes, R., 1986, Concept Study Proposal for an Asteroseismology 
Explorer, Univ. Calif. San Diego.

Noyes, R.W. and Rhodes, E.J. (eds.) 1984, Report of NASA Science Working Group on the Study of Solar Oscillations from Space," NASA, JPL.

Praderie, F., Mangeney, A., Lemaire, Ph., Puget, P. 1986, these proceedings.

Rhodes, E., Cacciani, A., Tomczyk, S., Ulrich, R., Blamont, J., Howard, R., Dumont, P., and Smith, E. 1984, in Advances in Space Research, 4, 103.

Rhodes, E., Tomczyk, S., and Cacciani, A. 1986, these proceedings.

Soderblom, D. 1986, these proceedings.

Willson, R.C. 1979, App. Optics, 18, 179.

Willson, R.C. and Hudson, H.S. 1981, Astrophys. J., 244, L185.

Woodard, M.F. and Noyes, R.W. 1985, Nature, 318, p. 449.

Woodard, M.F. and Noyes, R.W. 1986, these proceedings. 